02

\title{
Моделирование детектора зарядовых состояний релятивистских многозарядных ионов
}

\author{
() В.С. Малышевский, Г.В. Фомин, И.А. Иванова
}

Южный федеральный университет, Ростов-на-Дону

E-mail: vsmalyshevsky@sfedu.ru

Поступило в Редакцию 15 июля 2016 г.

Анализируется способ идентификации зарядовых распределений релятивистских многозарядных ионов, заключающийся в регистрации углового распределения черенковского излучения ионов с предварительным разделением по скоростям ионов с разными зарядами во внешней мишени с большим зарядовым числом. В результате при влете пучка ионов в черенковский радиатор разные заряды будут излучать под разными углами к направлению движения ионов, а интенсивность излучения будет пропорциональна доле ионов с заданным зарядом в пучке.

DOI: 10.21883/PJTF.2017.01.44090.16411

В экспериментах по взаимодействию тяжелых многозарядных ионов с веществом почти всегда необходимо не только измерять энергию частиц, но и идентифицировать их зарядовые состояния. Распределение по зарядовым состояниям особенно важно при формировании пучков многозарядных ионов для тех или иных целей. Проблема надежной идентификации частиц особенно остро стоит в физике релятивистских многозарядных ионов. Использование для этих целей обычного магнитного анализа [1] связано с необходимостью получения интенсивных магнитных полей, особенно в области ультрарелятивистских скоростей ионов, и рядом других технических трудностей. Аналогичными недостатками обладает и метод, основанный на измерении кривой Брэгга [2], который затруднительно применять в области релятивистских и ультрарелятивистских энергий ионов. Метод идентификации на основе измерений удельных потерь энергии и полной энергии (так называемый $\Delta E-E$-метод [3]) ограничен, во-первых, сверху по значению массового числа ионов $A<20$, а во-вторых, эффективен только в 
области сравнительно небольших энергий ионов. В недавней работе [4] было показано, что торможение релятивистских многозарядных ионов в черенковском радиаторе приводит к уширению полос черенковского излучения, которое также можно использовать для идентификации заряда пролетающего через радиатор иона.

Ниже мы покажем, что сочетание метода удельных потерь энергии и черенковского излучения позволит определить распределение по зарядовым состояниям в пучке релятивистских и ультрарелятивистских ионов. Если в исходном пучке ионов имеются частицы с разным значением заряда, то для идентификации их распределения с использованием черенковского излучения необходимо обеспечить разброс по скоростям ионов с разными зарядами. Это, конечно, происходит и в самом черенковском радиаторе, поскольку удельные потери энергии пропорциональны квадрату заряда ионов $Z_{i}$. Однако для более эффективного разделения зарядов по скоростям желательно использовать внешнюю мишень с большим зарядовым числом $Z_{t}$, так как удельные потери энергии пропорциональны заряду атомов мишени. После прохождения внешней мишени пучок ионов будет содержать ионы разных зарядов, движущихся с разными скоростями. При влете такого пучка в черенковский радиатор заряды будут излучать под разными углами относительно направления движения иона. Причем большему заряду будет соответствовать меньший угол, а интенсивность излучения будет пропорциональна доле ионов с заданным зарядом в пучке. Регистрация углового распределения возникающего излучения позволит определить распределение по зарядовым состояниям (полагаем, скорости ионов с разными зарядами $\beta_{i}=v_{i} / c$ таковы, что порог черенковского излучения выполнен).

Понятно, что малоугловое многократное рассеяние и изменение зарядов ионов как в мишени, так и в радиаторе будут влиять на корректность идентификации заряда и соответствующей доли ионов. Влияние этих факторов мы оценим ниже, а пока рассмотрим все процессы, пренебрегая многократным рассеянием и процессами потери или подхвата ионом электронов мишени.

Удельные потери кинетической энергии релятивистских ионов будем оценивать по известному соотношению Бете-Блоха

$$
\frac{d T_{i}}{d x}=-\frac{4 \pi n_{e} Z_{i}^{2} e^{4}}{m_{e} c^{2} \beta_{i}^{2}}\left[\ln \frac{2 m_{e} c^{2} \beta_{i}^{2}}{I}-\ln \left(1-\beta_{i}^{2}\right)-\beta_{i}^{2}\right],
$$

где $T_{i}=M c^{2}\left[\left(1-\beta_{i}^{2}\right)^{-1 / 2}-1\right]-$ кинетическая энергия иона, $M-$ масса иона, $n_{e}, m_{e}$ - концентрация и масса электронов мишени, $I-$ 


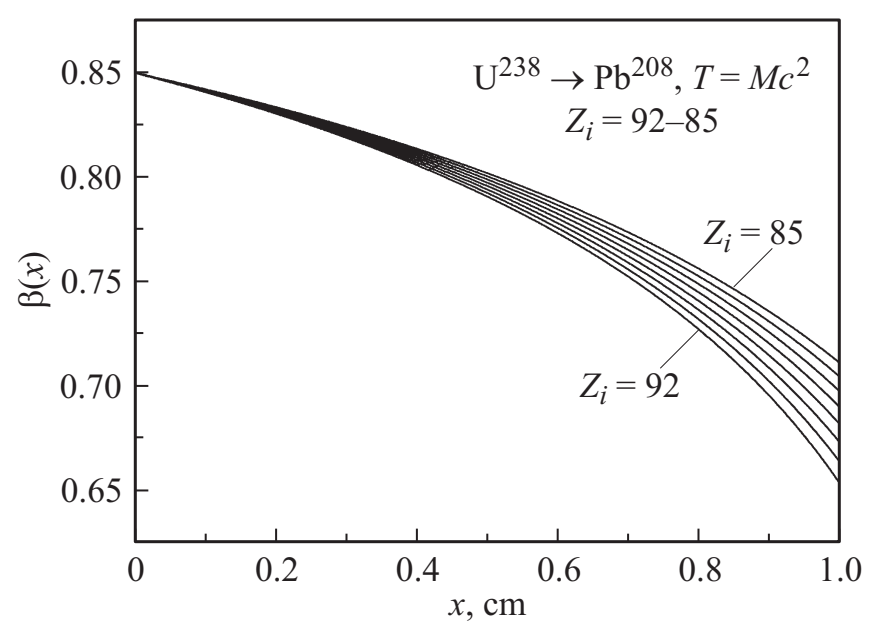

Рис. 1. Разброс скоростей ионов $\mathrm{U}^{238}$ с разными зарядами при начальной кинетической энергии, равной энергии покоя, после прохождения мишени $\mathrm{Pb}^{208}$ разной толщины.

средний потенциал ионизации атомов мишени, который можно оценить как $13.6 Z_{t}(\mathrm{eV})$. На рис. 1 показан пример расчета разброса скоростей согласно уравнению (1) релятивистских ионов $\mathrm{U}^{238} \mathrm{c}$ разными зарядами после прохождения мишени $\mathrm{Pb}^{208}$ разной толщины при начальной кинетической энергии, равной энергии покоя $T_{i}=M c^{2}$.

Расчет выхода черенковского излучения из радиатора конечной толщины $L$ можно провести методом, изложенным в [5] и модифицированным в [6] на случай изменения заряда иона в радиатоpe. А именно, электромагнитные поля в вакууме и в радиаторе находятся из условия непрерывности нормальной и тангенциальной компонент поля на границах раздела. Спектрально-угловая плотность излучения может быть тогда найдена традиционным путем расчета потока вектора Пойнтинга через удаленную поверхность. Как было отмечено выше, будем полагать, что толщина радиатора $L$ такова, что можно пренебречь торможением иона и считать его скорость постоянной. Такой способ позволяет описывать как возникающее излучение до порога возникновения черенковского излучения, так и когерентное излучение при выполнении условия $\beta_{i} \sqrt{\varepsilon^{\prime}}>1$,

Письма в ЖТФ, 2017, том 43, вып. 1 

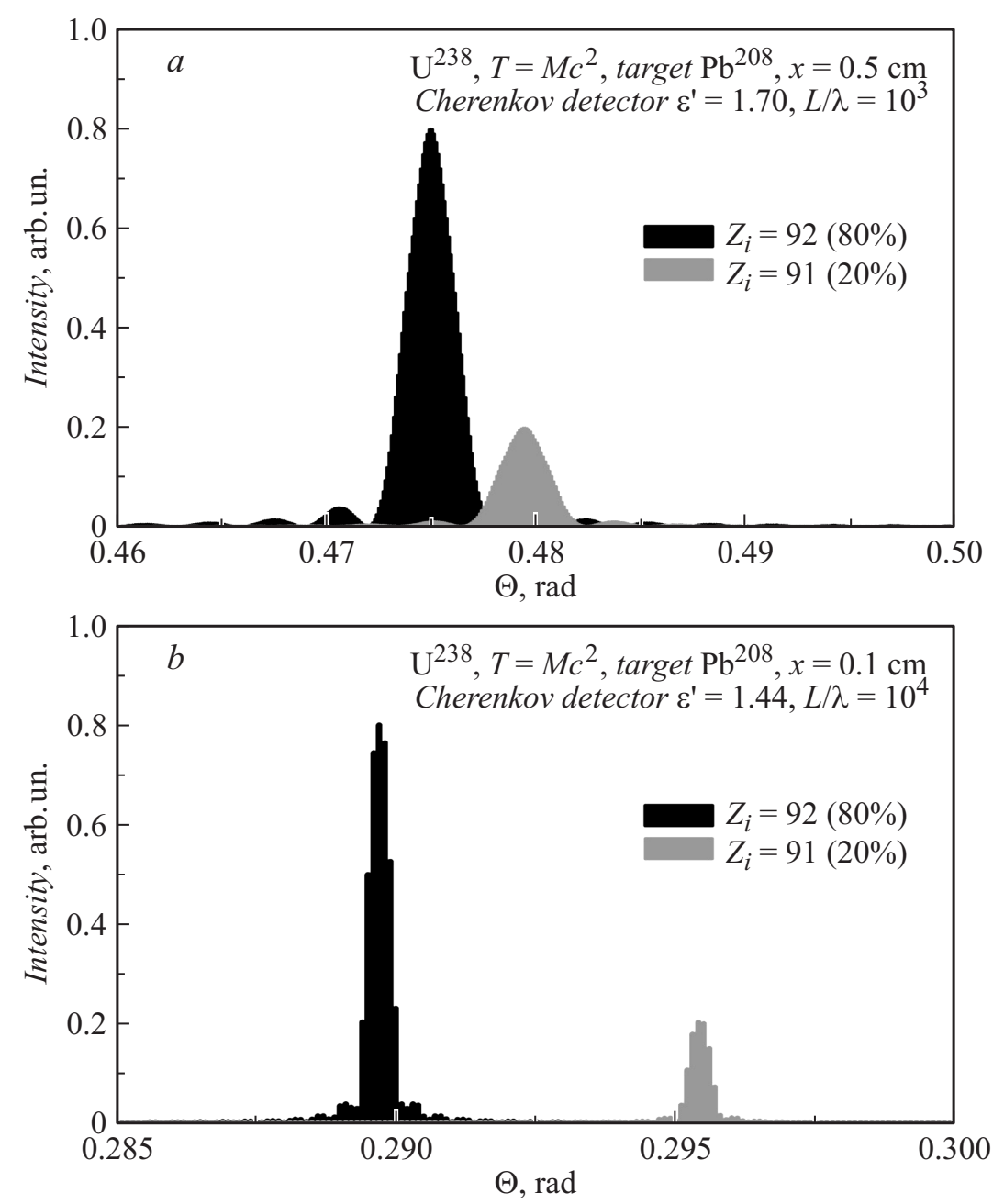

Рис. 2. Результаты расчетов черенковского излучения ионов $\mathrm{U}^{238}$, прошедших внешнюю мишень $\mathrm{Pb}^{208}$ толщиной $0.5 \mathrm{~cm}(a)$ и $0.1 \mathrm{~cm}(b)$. Предполагалось, что в исходном пучке содержится 80\% ионов с зарядом 92 и $20 \%$ с зарядом 91 . Действительная часть диэлектрической проницаемости радиатора $\varepsilon^{\prime}=1.7(a)$ и $1.44(b)$, толщина радиатора выбрана равной $10^{3}(a)$ и $10^{4}(b)$ длинам волн генерируемого излучения.

Письма в ЖТФ, 2017, том 43, вып. 1 
где $\varepsilon^{\prime}-$ действительная часть диэлектрической проницаемости радиатора.

Аналитические решения соответствующей системы уравнений Максвелла достаточно громоздки (см., например, [6]), и приводить их не будем. Однако численные расчеты с использованием аналитических формул можно легко сделать. Разработанный нами интерфейс позволяет в интерактивном режиме моделировать угловое распределение возникающего излучения с вариацией различных параметров: выбором скорости и заряда иона, толщины радиатора, углового диапазона излучения (вперед или назад), действительной и мнимой части диэлектрической проницаемости.

Некоторые результаты расчетов черенковского излучения ионов $\mathrm{U}^{238}$, прошедших внешнюю мишень $\mathrm{Pb}^{208}$ разной толщины, показаны на рис. 2, $a$ и $b$. Как видно, угловая дистанция между пиками достаточна для их уверенного разрешения. Ширина пиков значительно уменьшается при использовании более толстых радиаторов черенковского излучения.

Теперь оценим, как повлияют на результат, показанный на рис. 2, такие факторы, как малоугловое многократное рассеяние ионов, а также изменение зарядового состояния иона вследствие процессов потери или подхвата электронов в мишени и радиаторе. Среднеквадратичный угол многократного рассеяния во внешней мишени на длине пробега $x$ можно оценить из соотношения

$$
\left\langle\vartheta^{2}\right\rangle \approx 2 \pi n_{t}\left(Z_{i} Z_{t} e^{2} / T_{i}\right)^{2} x
$$

где $n_{t}-$ концентрация атомов мишени. Легко убедиться, что при использованных в расчетах параметрах, а именно кинетической энергии $T_{i}=M c^{2}$ ионов $\mathrm{U}^{238}$ и толщине внешней мишени $\mathrm{Pb}^{208}$ $x=0.5 \mathrm{~cm}$, среднеквадратичный угол рассеяния составит величину $\sqrt{\langle\vartheta\rangle} \approx 10^{-3} \mathrm{rad}$. Понятно, что такой маленький разброс по углам не может повлиять на разделение пиков черенковского излучения, расстояние между которыми на порядок больше.

Далее, сечение ионизации внутренних оболочек тяжелых ионов релятивистскими электронами так же, как и сечение радиационного захвата электронов мишени, составляет величину порядка 10-30 barn (см., например, [7-9]). Следовательно, вероятность изменения зарядового состояния ионов $\mathrm{U}^{238}$ с энергией $T_{i}=M c^{2}$ в мишени $\mathrm{Pb}^{208}$ толщиной $x=0.5 \mathrm{~cm}$ будет не более $5 \%$. Таким образом, проведенный анализ

5 Письма в ЖТФ, 2017, том 43, вып. 1 
показывает, что использование комбинированного метода удельных потерь энергии и черенковского излучения позволит надежно проводить идентификацию распределения тяжелых релятивистских ионов по зарядовым состояниям.

Работа поддержана грантом Южного федерального университета (проект № 213.01-07.2014/13 ПЧВГ).

\section{Список литературы}

[1] Курашов А.А. Идентификация ионизирующих излучений средних и низких энергий. М.: Атомиздат, 1979.

[2] Gruhn C.R. et al. // Nucl. Instr. Meth. 1982. V. 196. P. 33.

[3] Surface and Thin Film Analysis. Ed. by Gernot Friedbacher and Henning Bubert. Wiley-VCH Verlag GmbH \& Co. KGA. 2011. 532 p.

[4] Богданов О.В., Фикс Е.И., Пивоваров Ю.Л. // ЖЭТФ. 2012. V. 142. Р. 442.

[5] Тер-Микаелян М.Л. Влияние среды на электромагнитные процессы при высоких энергиях. Ереван: Изд-во АН Арм. ССР, 1969. С. 210.

[6] Мальшевский В.С., Иванова И.А., Фомин Г.В. // ЖЭТФ. 2016. Т. 149. С. 243.

[7] Бейгман И.Л., Толстихина И.Ю., Шевелько В.П. // ЖТФ. 2008. Т. 78. С. 15.

[8] Nelson Claytor et al. // Phys. Rev. Lett. 1988. V. 61. P. 2081.

[9] Anholt R., Staller Ch., Molitoris J.D. et al. // Phys. Rev. A. 1986. V. 33. P. 2270. 